\title{
Gene expression profile analysis of the progression of carotid atherosclerotic plaques
}

\author{
WANGMI LIU ${ }^{1}$, YANXIN ZHAO ${ }^{2}$ and JIAYAN WU ${ }^{2}$ \\ ${ }^{1}$ Department of Orthopedics, The Second Affiliated Hospital, Zhejiang University School of Medicine, \\ Hangzhou, Zhejiang 310009; ${ }^{2}$ Department of Neurology, Shanghai Tenth People's Hospital, \\ School of Medicine, Tongji University, Shanghai 200032, P.R. China
}

Received May 6, 2016; Accepted March 30, 2017

DOI: $10.3892 / \mathrm{mmr} .2018 .8575$

\begin{abstract}
The present study aimed to examine the universal gene expression signature and the underlying molecular mechanisms involved in the progression of carotid atherosclerotic plaques. The gene expression dataset, GSE28829, containing 13 early and 16 advanced carotid atherosclerotic plaques was selected for analysis. The differentially expressed genes (DEGs) were identified and analyzed using bioinformatics analyses, including cluster analysis, Gene Ontology (GO) and pathway enrichment analyses. Finally, a protein-protein interaction (PPI) was constructed and analyzed. A total of 515 downregulated and 243 downregulated DEGs were identified. The cluster analysis revealed two separate two groups. In addition, the GO terms enriched by the upregulated DEGs were associated with immune response, and the downregulated DEGs were associated with cell adhesion. The upregulated DEGs were enriched in pathways associated with signaling in the immune system, and the downregulated DEGs were enriched in pathways associated with muscle contraction. In the PPI network analysis, ITGAM and ACTN2 had the highest decrees of connectivity in the upregulated and downregulated DEGs, respectively. These findings suggested that deregulation of the immune system and smooth muscle cell cytoskeleton accelerates the progression of carotid atherosclerotic plaques. The DEGs identified may offer potential in the prevention and treatment of atherosclerosis in the carotid artery.
\end{abstract}

\section{Introduction}

Ischemic stroke (IS), one of the major causes of mortality and disability, and comprises $>80 \%$ of stroke cases, compared with hemorrhagic stroke accounting for the remainder (1).

Correspondence to: Dr Jiayan Wu, Department of Neurology, Shanghai Tenth People's Hospital, School of Medicine, Tongji University, 301 Yanchangzhong Road, Shanghai 200032, P.R. China E-mail: lcyxwjy@163.com

Key words: carotid atherosclerotic plaque, differentially expressed genes, functional enrichment analysis, microarray
Transient or permanent reduction in middle cerebral artery flow leads to IS, which is primarily caused by an embolus or local thrombosis (2). As carotid atherosclerotic plaques represent one of the sources of emboli, they are associated with $\sim 15 \%$ of IS cases and future stroke development (3), in addition to predisposing patients to fainting and syncope. High socio-economic costs, persistent neurological impairment and physical disabilities are usually inevitable when IS occurs in an individual due to the narrow therapeutic time window (4). Therefore, there is motivation to improve the prevention of IS through interference with etiological factors, including carotid atherosclerotic plaques, rather than treating IS passively.

Carotid atherosclerotic plaques are derived from atherosclerosis, which is a complex process characterized by inflammation of the vascular wall and accumulation of lipids (5). From a pathophysiological point of view, plaque composition alters dynamically during the course of atherosclerosis. Therefore, there are a number of differences between early and advanced plaques, including the thickness of the fibrous cap, the size of the necrotic core and intraplaque hemorrhage (6). The advanced plaque is more likely to rupture, leading to IS (7); therefore, it is a reasonable approach to prevent the progression of carotid atherosclerotic plaques. Identifying the factors associated with progression can provide a foundation for further manipulation.

Several studies have suggested factors involved in plaque progression from different points of view and have led to different decisions. For example, endothelial dysfunction due to injury initiates atherosclerosis (8). High plasma concentrations of low-density lipoprotein and homocysteine promote arterial inflammation, leading to the aggregation of inflammatory cells under the endothelium, including macrophages and lymphocytes (9). Several cytokines, particularly chemokines have been identified as causal factors in plaque progression (10). However, as carotid atherosclerotic plaque formation is a multifactorial disease, a comprehensive and multi-dimensional perspective is required. Therefore, it is more meaningful to analyze the progression at a higher level, including the biological processes and pathways involved, rather than determining the fold changes of specific genes or behavioral changes of cells. 
Advances in molecular genetics have enabled the simultaneous screening of differentially expressed genes (DEGs) between samples using microarrays. Therefore, information regarding how to understand the complex nature of carotid atherosclerotic plaques has been obtained, which is based on data on the temporal and spatial variation of key genes, and the use of bioinformatics methods to perform systematic analysis (11-15). Regarding the available relevant datasets in the Gene Expression Omnibus (GEO), the present study examined the gene expression dataset, GSE28829, which includes gene expression profiles of early and advanced carotid atherosclerotic plaques. Through in-depth analysis of the dataset, the present study aimed to provide a comprehensive perspective for understanding the molecular mechanism underlying carotid atherosclerotic plaques, and for identifying novel therapeutic targets for the prevention and treatment of carotid atherosclerotic plaques.

\section{Materials and methods}

Microarray datasets. The GSE28829 dataset was downloaded from the GEO (https://www.ncbi.nlm.nih.gov/geo/) for analysis in the present study. The gene expression profiles of 16 advanced and 13 early carotid atherosclerotic plaques from autopsy were determined using the Affymetrix Human Genome U133 Plus 2.0 Array (Affymetrix, Inc., Santa Clara, CA, USA). As it not ethical or possible to obtain early carotid atherosclerotic plaques in clinical practice, these specimens were collected during autopsy and stored at the Maastricht Pathology Tissue Collection (Maastricht, the Netherlands). An advanced carotid atherosclerotic plaque was defined as a plaque with a thin or thick fibrous cap, whereas an early carotid atherosclerotic plaque was identified as intimal thickening and intimal xanthoma.

Data preprocessing. The gene expression profiles were assessed using Expression Console 1.4.1.46 (Affymetrix, Inc.). Data preprocessing, including background adjustment, normalization and log transformation of the values, was performed using the robust multiarray average method (12). In doing so, the probe-level data were converted to gene expression values. When several probes corresponded to one gene symbol, the mean of the probe-level data was taken to represent the gene expression value.

Differential expression analysis and clustering. An unpaired t-test was used to identify the DEGs between the two groups using R (version 3.2.3; Statistics Department of the University of Auckland). To avoid an excess false positive results due to multi-test problems, the raw P-values were adjusted into the false discovery rate (FDR) using the Benjamin and Hochberg method (16). An FDR $<0.05$ and a $\log _{2}$ Fold Change (FC) $1>0.58$ (17) were used as the cut-off criterion to identify the final DEGs. Cluster analysis was then used to group the cases into clusters according to the DEGs using $\mathrm{R}$ (version 3.2.3; Statistics Department of the University of Auckland). The cluster analysis was able to group the cases based on the similarity in their gene expression profiles. The cases assigned to the same cluster were more closely associated with one another, compared with cases assigned to separate clusters.

GO and pathway enrichment analyses. GO (18), a tool for the unification of biology is comprised of biological process, molecular function and cellular component. The Reactome pathway database is a curated, peer-reviewed pathway database of human biological processes, which is used for the classification of correlating gene sets into their respective pathways (19). To analyze the DEGs at the functional level, GO and Reactome pathway enrichment analyses were performed using the Database for Annotation, Visualization and Integrated Discovery (DAVID; https://david.ncifcrf.gov) online tool to obtain the enriched GO terms and pathways via a clustering algorithm (20). $\mathrm{P}<0.05$ was set as the threshold value.

PPI network construction. In the present study, the online tool of the Search Tool for the Retrieval of Interacting Genes (STRING) database 10.0 was used to screen the PPIs of the DEGs, with a required confidence (combined score) $>0.4$ (21). To assist in visualizing the biological networks and integrating the data generated by the STRING database, the PPI networks were constructed and visualized using Cytoscape (version 3.3.0; http://cytoscape.org/) (22). As the majority of the networks were scale-free, the hub genes were then selected with a connectivity degree $>15$ (23).

\section{Results}

Data preprocessing and identification of DEGs. The expression distribution characteristics of the dataset prior to and following data pretreatment are shown in Fig. 1A and B. Their median values were almost on a straight line, indicating that the raw data were normalized successfully. The DEGs, including the upregulated and downregulated genes, were identified from the two groups with an $\mathrm{FDR}<0.05$ and a $\mid \log _{2} \mathrm{FCl}>0.58$. As a result, a total of 758 DEGs were obtained following data processing. Among these, there were 515 upregulated genes and 243 downregulated genes.

Cluster analysis. The clustering of the DEGs showed that the signature of the majority of the advanced carotid atherosclerotic plaques resembled one another, rather than one of the early plaques, and vice versa (Fig. 2). There were notable differences between the advanced and early carotid atherosclerotic plaques according to their gene expression profiles. However, there was one advanced carotid atherosclerotic plaque clustered in the early group, and one early carotid atherosclerotic plaque clustered in the advanced group, suggesting that the gene expression profiles of those two patients were different from their own group.

GO enrichment analyses. The DEGs were analyzed using the DAVID tool and categorized into the three major terms in GO, the biological process, molecular function and cellular component. The top three GO terms of the three categories are shown in Fig. 3. The enriched terms of the upregulated genes were significantly associated with immune response, IgG binding and plasma membrane in the three categories, respectively (Fig. 3A). By contrast, the enriched terms identified among 
A

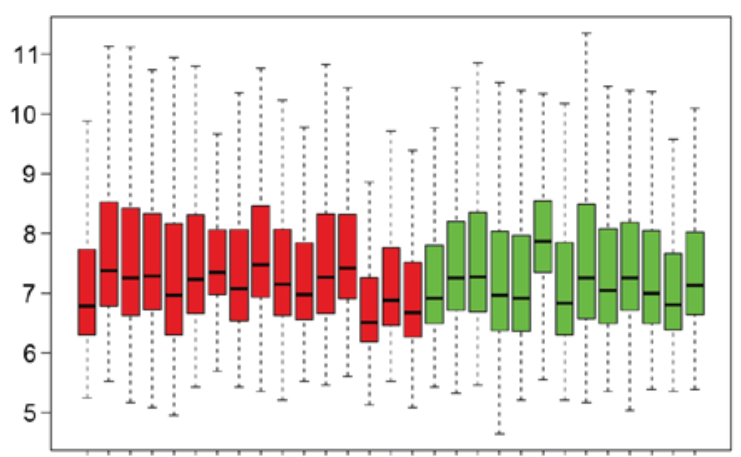

B

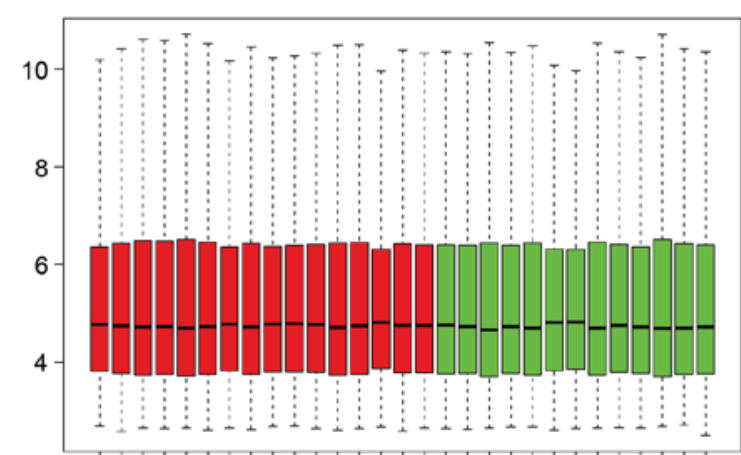

Figure 1. Expression distribution characteristics of the dataset. Dataset (A) prior to and (B) following data preprocessing. Each box contains a black line representing the median gene expression level, which was almost at the same level across all samples. Red indicates advanced carotid atherosclerotic plaques; green indicates early carotid atherosclerotic plaques.

the downregulated genes were associated with cell adhesion, actin binding and contractile fiber in the three categories, respectively (Fig. 3B).

Pathway enrichment analysis. To examine the biological pathways associated with the DEGs in the progression of carotid atherosclerotic plaques, the DAVID tool was also used. The upregulated genes were intrinsically linked with signaling in immune system, hemostasis and integrin cell surface interactions (Fig. 3C). By contrast, the downregulated genes were significantly associated with muscle contraction only (Fig. 3C).

PPI network construction. The PPI networks with the hub genes possessing a connectivity degree of $>15$ were constructed using Cytoscape software, based on the results from the analysis using the STRING database. Networks with 120 and 101 nodes were obtained using the upregulated and downregulated genes, respectively (Fig. 4A and B). Within a PPI network, each node indicates a gene, whereas the edges represent the interactions between nodes. The degree represents the number of edges linked to a specific node. Therefore, nodes with a high degree are defined as hub genes, which assume important biological functions. A total of 13 hub genes were selected from the upregulated PPI network, which included ITGAM, PTPRC and APOE, which had connectivity degrees of 37, 27 and 26, respectively. From the downregulated PPI network, two hub genes, ACTN2 and
$V C L$, with respective connectivity degrees of 19 and 17 , were identified.

\section{Discussion}

A number of previous studies have used the GSE28829 dataset to examine the DEGs involved in the progression of carotid atherosclerotic plaques $(12,14)$. However, one of these two studies bridged the gap between the gene expression of M1/M2 macrophage phenotypes and antioxidant genes in atherosclerosis, which only included a subset of the dataset (14). The other study adopted a stricter standard, compared with that used in the present study, to judge the DEGs, resulting in relatively fewer results returned from the functional and pathway enrichment analysis, compared with the present study (12). As carotid atherosclerotic plaques formation is a multifactorial disease, identifying additional potential biological processes and pathways offers potential in palliation or curing of the disease. It can be difficult to directly counteract the effects of each key gene; therefore, it is necessary to abate the effects through the biological processes and pathways enriched by these key genes. Therefore, the results of the present study provide a more comprehensive perspective towards understanding the molecular mechanism and towards discovering novel therapeutic targets in this field.

In the present study, 758 DEGs were obtained following analysis of the microarray data deposited in the GSE28829 dataset. This gene expression signature indicated that the expression of the selected genes was altered during the progression of carotid atherosclerotic plaque formation. Although expression analyses from the dataset cannot confirm a causal association between altered gene expression and plaque state, there is merit in performing an integrative systematic bioinformatics approach, including functional and pathway analyses, and in the construction of a PPI network based on DEGs. This may assist in understanding the molecular mechanisms underlying carotid atherosclerotic plaques at higher levels, providing an insight into the prevention and treatment of carotid atherosclerotic plaques.

The results of the cluster analysis suggested marked differences between the advanced and early carotid atherosclerotic plaques, and also indicated that the selected genes were associated with disease progression. Of note, the samples did not perfectly group together in the two groups in the heatmap as expected. There are several factors, which may have contributed to the presence of one advanced plaque within the early plaque group and one early plaque within the advanced plaque group. Experimental variance, including RNA isolation, and biological variance, including incorrect classification of samples during autopsy, may affect the interpretation of datasets (24). However, it is possible that the group inconsistency was due to other genes not considered in the present study.

The results of the functional analysis from $\mathrm{GO}$ enabled the translation of DEGs into the underlying biological mechanism. In the present study, the enriched GO terms from the upregulated DEGs were primarily associated with immune response and IgG binding, which occurred in the plasma membrane. This suggested the important effects of immune cells on the initiation and progression of the 


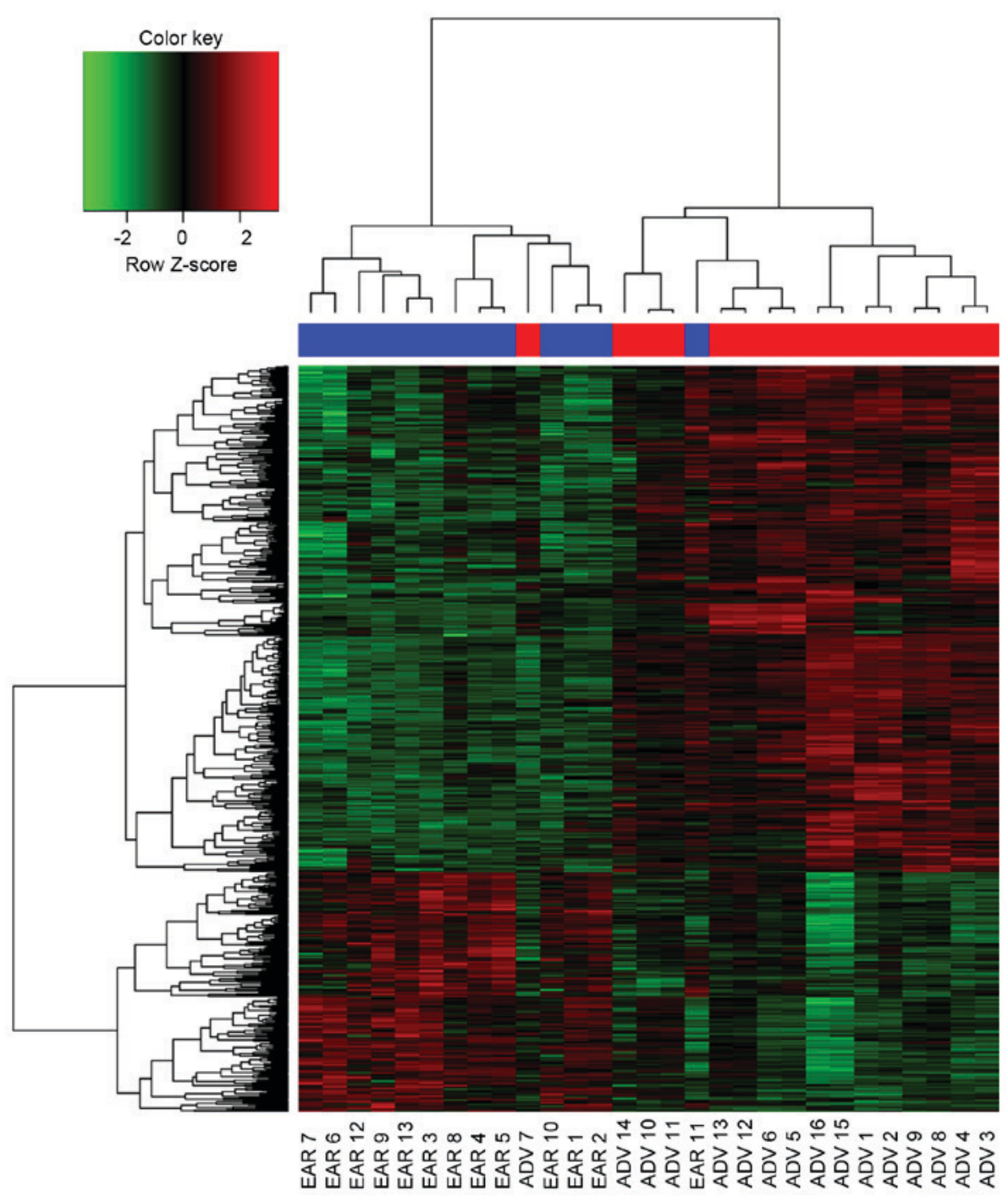

Figure 2. Heatmap of the DEGs between the two groups and hierarchical cluster analysis. ADV 7 was clustered in the early group, whereas EAR 11 was clustered in the advanced group. Red indicates upregulated DEGs; green indicates downregulated DEGs. ADV, advanced carotid atherosclerotic plaque (red at the top); EAR, early carotid atherosclerotic plaque (blue at the top); DEGs, differentially expressed genes.

atherosclerotic process (25). The enriched GO terms from the downregulated DEGs were primarily linked to cell adhesion and actin binding, which existed in the contractile fiber. This is concordant with the suggestion that dysregulated cytoskeletal proteins can abate their ability to promote endothelial repair through alterations in cell shape and through regulation of cell migration following wounding of the endothelium (26). Therefore, the upregulated and downregulated DEGs acted in concert to accelerate the atherosclerotic process.

Pathway analysis using the Reactome database identifies differential expression patterns based on gene groups rather than individual genes; in cases where an individual genes shows subtle biological function or property changes, they are omitted by the usual individual gene analysis (27). The results of the present study showed that the enriched pathways from the upregulated genes were predominantly associated with signaling in immune system, whereas the downregulated genes were associated with muscle contraction. These results overlapped with the GO enrichment analyses, therefore, the data supported the findings of previous studies, which reported immunity to be involved in the process of the initiation and progression of carotid atherosclerotic plaques intertwined with blood vessel dysfunction $(28,29)$.

PPI networks are critical for understanding underlying mechanisms as these vital activities are ascribed to interactions between proteins to form a signal transduction network system when confronted with external and internal environmental stimuli. As several vital activities are associated with the combination and dissociation of proteins, hub genes are important in this process. The top hub genes identified in the present study were ITGAM and ACTN2 from the upregulated and downregulated genes, respectively. ITGAM encodes the integrin $\alpha \mathrm{M}$ chain. An $\alpha$ chain and a $\beta$ chain combine to form a heterodimeric integral membrane protein, integrin, which mediates the adhesion of cells to extracellular matrix-associated proteins. The $\alpha \mathrm{M} \beta 2$ integrin is involved in the adherence of neutrophils and monocytes to stimulated endothelium in cases of inflammation and thrombosis (30), and also in CD40L-mediated inflammation during atherogenesis as an alternative pathway (31). ACTN2 is identical to one of the key interaction nodes reported by Perisic et al (13), 
A

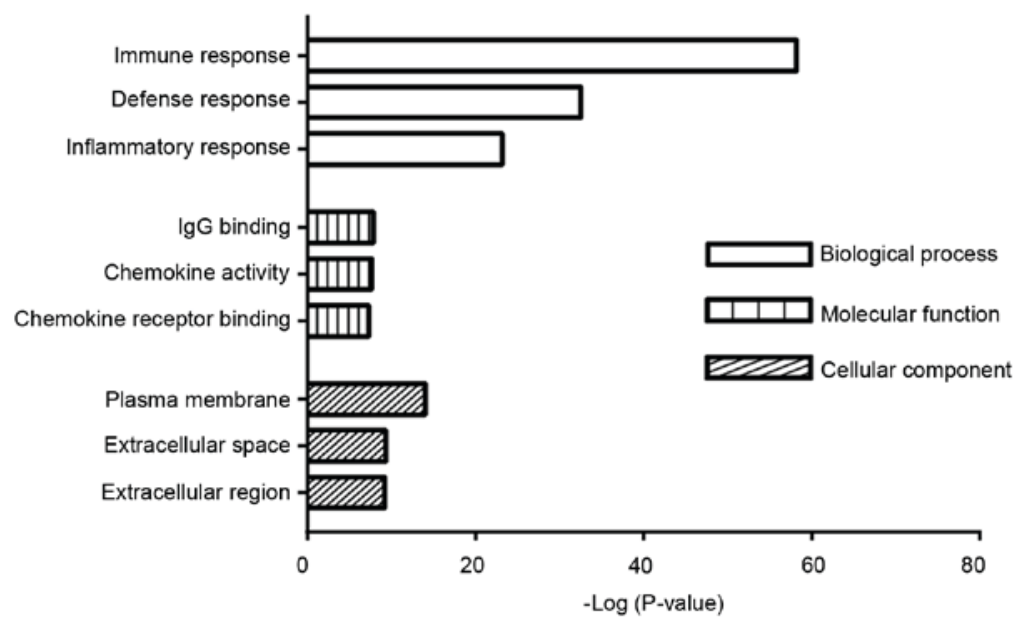

B

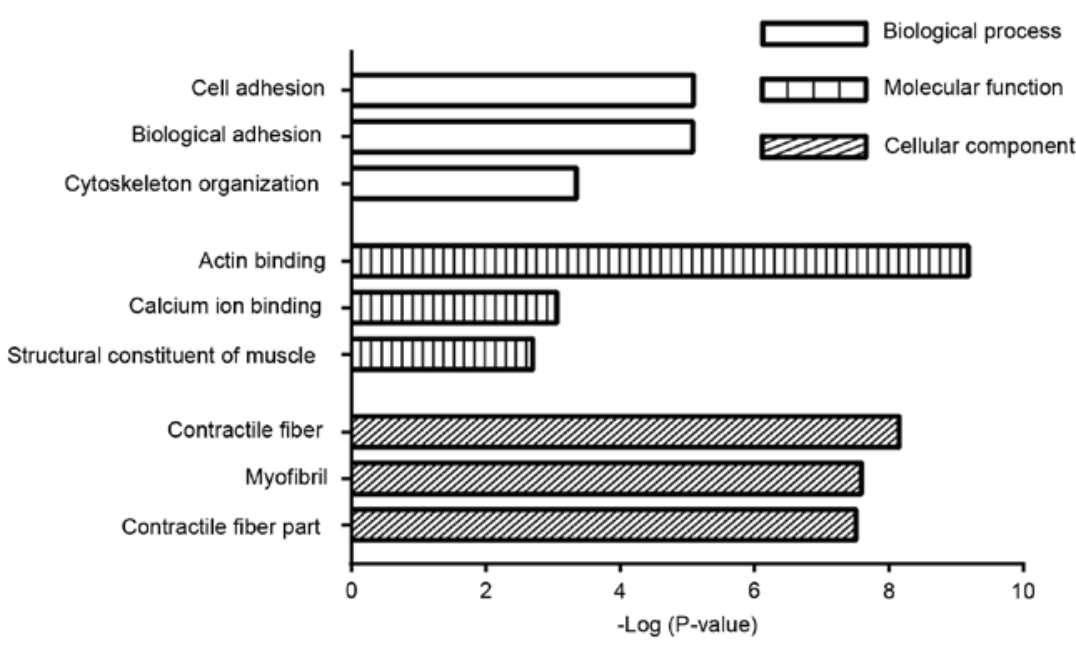

C

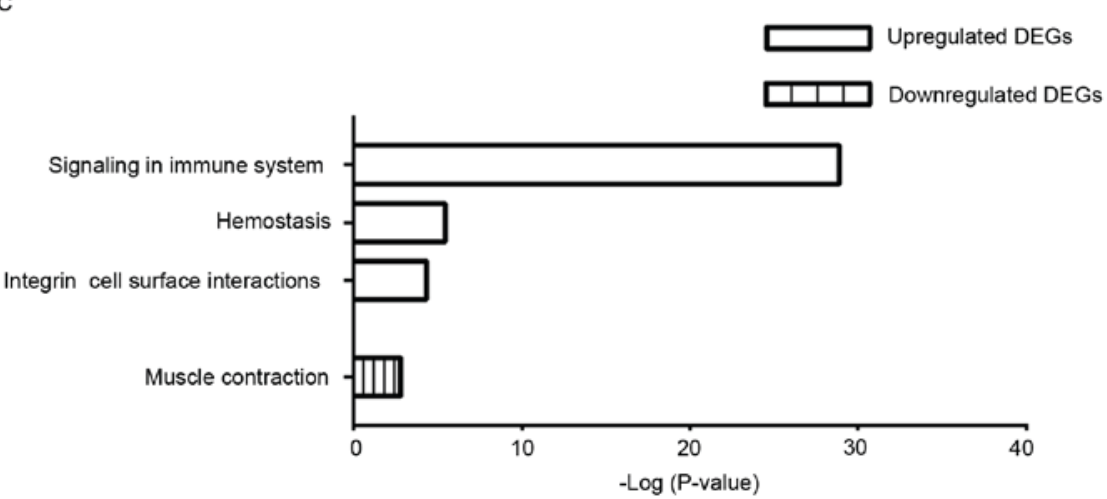

Figure 3. Functional and pathway analyses using the Database for Annotation, Visualization and Integrated Discovery. (A) Enriched GO terms from the upregulated DEGs. (B) Enriched GO terms from the downregulated DEGs. (C) Enriched Reactome pathways from the upregulated and downregulated DEGs. GO, Gene Ontology; DEGs, differentially expressed genes.

which encodes a cytoskeletal protein belonging to the spectrin gene superfamily. This actin-binding protein has multiple roles in different cell types. In smooth muscle, the protein assists in anchoring the myofibrillar actin filaments to the Z-disc and analogous dense bodies. Together with $V C L$, which also encodes a cytoskeletal protein, this cytoskeletal deregulation supports the hypothesis that medial vascular smooth muscle cells switch from a contractile to a synthetic phenotype during the development of atherosclerosis at the molecular level (32). This is in accordance with the above-mentioned dysregulated muscle contraction identified from the pathway analyses of the downregulated DEGs.

There were a number of limitations in the present study. The samples were collected from autopsy, affecting the quality of isolated RNA and downstream analysis of the dataset. In addition, the results from the array and bioinformatics analyses require further biological experiments. Therefore, 
A

\begin{tabular}{|c|c|c|c|c|c|c|c|c|}
\hline HLA-DQB1 & C1QB & VCAM1 & MAFB & CCL4 & CCL3 & ACP5 & CD86 & THEMIS2 \\
\hline LY86 & HLA-DRBS & TLR2 & ITGAM & RAC2 & CD163 & IER3 & LGMN & IQGAP2 \\
\hline$M S 4 A 6 A$ & $\mathrm{ABCA} 1$ & LY96 & HCLS1 & UCP2 & CCL8 & FPR3 & APOC1 & PLA2G7 \\
\hline $\mathrm{BCL} 2 \mathrm{~A} 1$ & IL7R & MMP9 & APOE & LIPA & HLA-DRA & IGJ & PLTP & TREM2 \\
\hline HLA-DMB & $\mathrm{IF} \mid 30$ & HTR2B & JRL1B & C3AR1 & PLA2G2A & IRF8 & LYN & RGS1 \\
\hline FERMT3 & CAPG & CCR1 & DX5AP & TNFAIP3 & $\mathrm{P} 2 \mathrm{R} \times 7$ & CCL18 & CXCL16 & CD83 \\
\hline SPP1 & & & C1QA & & IL10RA & & $\mathrm{HP}$ & AlF1 \\
\hline CXCR4 & & CXCL14 & MYO1F & LAPTM5 & $\mathrm{CD} 37$ & MARCKS & FCGR3A & FCGR1B \\
\hline PTPRC & CD68 & $\mathrm{HCK}$ & ALOX5 & ITGB2 & CD14 & $\mathrm{co}$ & ADRA2A & CTSB \\
\hline $\mathrm{CFI}$ & BCAT1 & FCGR1A & HLA-DPA1 & EDN1 & LCP1 & INE2 & FCGR2A & TYROBP \\
\hline CLEC5A & $\mathrm{C} 1 \mathrm{QC}$ & MPEG1 & SERPINA 1 & CHI3L1 & DAPK1 & HLA-DRB & F13A1 & $\mathrm{C} 4 \mathrm{~A}$ \\
\hline SLC1A3 & NgE2 & TREM1 & CD53 & COLEC 12 & CSF2RB & TNFSF13E & CXCL2 & TNC \\
\hline HLA-DPB1 & DAB2 & CSF1R & CTSS & FCER1G & FCGR2B & CCL19 & C5AR1 & SGK1 \\
\hline COR01A & MSR1 & HS3ST2 & & & & & & \\
\hline
\end{tabular}

B

\begin{tabular}{|c|c|c|c|c|c|c|c|c|}
\hline AMPH & RBM20 & PGR & FAT3 & FRK & EFHD1 & LGR6 & PLXNB1 & PIP5K1B \\
\hline PLCB4 & PDZRN4 & HSPB1 & HSPB8 & PALLD & COL4A5 & CNN1 & FHL5 & CNTN4 \\
\hline BAMBI & SLMAP & PHACTR 1 & INPP5A & ITGA9 & DMPK & KCNMB1 & YAP1 & SORT1 \\
\hline TPM2 & SVIL & CASQ2 & PKD2 & MPP7 & PFN2 & TMOD1 & $\mathrm{HADH}$ & MICAL2 \\
\hline FERMT2 & PDLIM7 & RRAS2 & MYBL1 & CFL2 & PP1R12B & SYNC & MYOCD & SPEG \\
\hline PLCL1 & ITGA7 & NPR1 & PTPLA & ACTN2 & $\mathrm{RHOB}$ & SMTN & RERG & PPP1R14A \\
\hline CAMK2G & ZNRF3 & ALDH1B1 & STK38L & JPH2 & OXCT1 & MYLK & MYO18B & DMD \\
\hline CRYAB & BAG2 & ROR1 & GPR125 & CKB & PYGB & PLN & BHMT2 & SORBS1 \\
\hline MAP2 & PPP1R1A & SYNM & AGTR1 & ARHGEF2 & ALDH1L1 & THRB & ACADL & CAB39L \\
\hline KCNMA1 & TES & LMOD1 & JAK2 & COL21A1 & PPP2R2B & BMPR1A & ANGPTL1 & ACTG2 \\
\hline MN1 & COL18A1 & FLNC & ATP1A2 & VCL & NEXN & PDZRN3 & MCAM & FABP3 \\
\hline RASSF3 & $\mathrm{PCDH} 7$ & & & & & & & \\
\hline
\end{tabular}

Figure 4. Constructed PPI network of the DEGs. (A) PPI network of the upregulated DEGs. (B) PPI network of the downregulated DEGs. The hub genes are shown as black boxes with white text. PPI, protein-protein interaction; DEGs, differentially expressed genes.

genetic and experimental investigations with strict protocols are required based on the results of the present study in the future.

In conclusion, based on comprehensive bioinformatics analyses of the dataset, the data obtained in the present study elucidated the DEGs, which are potentially associated with the common molecular mechanism underlying the progression of carotid atherosclerotic plaques. Furthermore, deregulation of the immune system and smooth muscle cell cytoskeleton were associated with the progression of carotid atherosclerotic plaques. These findings provide novel insights into the biological properties of carotid atherosclerotic plaques, and provide clues and direction for the prevention and treatment of atherosclerosis in the carotid artery. Further experiments are required in order to confirm these results.

\section{Acknowledgements}

The authors would like to thank Dr. Marco Manca for uploading data to the GEO database., as well as Professor Xueyuan Liu and Dr. Yanyan Zhao (Department of Neurology, 
Shanghai Tenth People's Hospital, School of Medicine, Tongji University, Shanghai, China) for revising the article.

\section{Funding}

No funding was received.

\section{Availability of data and materials}

The analyzed data sets generated during the study are available from the corresponding author on reasonable request.

\section{Authors' contributions}

JW conceived the study. WL and YZ performed the analysis and wrote the paper. All authors read and approved the manuscript.

\section{Ethics approval and consent to participate}

Not applicable.

\section{Consent for publication}

Not applicable.

\section{Competing interests}

All authors declared that they have no conflict of interest with regard to this work.

\section{References}

1. Dichgans M: Genetics of ischaemic stroke. Lancet Neurol 6: 149-161, 2007.

2. Dirnagl U, Iadecola C and Moskowitz MA: Pathobiology of ischaemic stroke: An integrated view. Trends Neurosci 22: 391-397, 1999.

3. Palm F, Dos Santos M, Urbanek C, Greulich M,Zimmer K, Safer A, Grau AJ and Becher H: Stroke seasonality associations with subtype etiology and laboratory results in the Ludwigshafen Stroke Study (LuSSt). Eur J Epidemiol 28: 373-381, 2013.

4. Schellinger PD, Kaste M and Hacke W: An update on thrombolytic therapy for acute stroke. Curr Opin Neurol 17: 69-77, 2004

5. Ross R: Atherosclerosis-an inflammatory disease. N Engl J Med 340: 115-126, 1999.

6. Teng Z, Sadat U, Brown AJ and Gillard JH: Plaque hemorrhage in carotid artery disease: Pathogenesis, clinical and biomechanical considerations. J Biomech 47: 847-858, 2014.

7. McNally JS, McLaughlin MS, Hinckley PJ, Treiman SM, Stoddard GJ, Parker DL and Treiman GS: Intraluminal thrombus, intraplaque hemorrhage, plaque thickness, and current smoking optimally predict carotid stroke. Stroke 46: 84-90, 2015.

8. Gimbrone MA Jr and García-Cardeña G: Endothelial cell dysfunction and the pathobiology of atherosclerosis. Circ Res 118: 620-636, 2016.

9. Luque MC, Gutierrez PS, Debbas V, Kalil J and Stolf BS: CD100 and plexins B2 and B1 mediate monocyte-endothelial cell adhesion and might take part in atherogenesis. Mol Immunol 67: 559-567, 2015.

10. Wong HS, Jaumouille V, Freeman SA, Doodnauth SA, Schlam D, Canton J, Mukovozov IM, Saric A, Grinstein S and Robinson LA Chemokine signaling enhances CD36 responsiveness toward oxidized low-density lipoproteins and accelerates foam cell formation. Cell Rep 14: 2859-2871, 2016.

11. Wang Z, Guo D, Yang B, Wang J, Wang R, Wang X and Zhang Q Integrated analysis of microarray data of atherosclerotic plaques: Modulation of the ubiquitin-proteasome system. PLoS One 9: e110288, 2014.
12. Wang J, Wei B, Cao S, Xu F, Chen W, Lin H, Du C and Sun Z: Identification by microarray technology of key genes involved in the progression of carotid atherosclerotic plaque. Genes Genet Syst 89: 253-258, 2014.

13. Perisic L, Aldi S, Sun Y, Folkersen L, Razuvaev A, Roy J, Lengquist M, Åkesson S, Wheelock CE, Maegdefessel L, et al: Gene expression signatures, pathways and networks in carotid atherosclerosis. J Intern Med 279: 293-308, 2016.

14. da Rocha RF, De Bastiani MA and Klamt F: Bioinformatics approach to evaluate differential gene expression of M1/M2 macrophage phenotypes and antioxidant genes in atherosclerosis. Cell Biochem Biophys 70: 831-839, 2014.

15. Cipollone F, Felicioni L, Sarzani R, Ucchino S, Spigonardo F, Mandolini C, Malatesta S, Bucci M, Mammarella C, Santovito D, et al: A unique microRNA signature associated with plaque instability in humans. Stroke 42: 2556-2563, 2011.

16. Reiner A, Yekutieli D and Benjamini Y: Identifying differentially expressed genes using false discovery rate controlling procedures. Bioinformatics 19: 368-375, 2003.

17. Sun J, Wen X, Jin F, Li Y, Hu J and Sun Y: Bioinformatics analyses of differentially expressed genes associated with bisphosphonate-related osteonecrosis of the jaw in patients with multiple myeloma. Onco Targets Ther 8: 2681-2688, 2015.

18. Ashburner M, Ball CA, Blake JA, Botstein D, Butler H, Cherry JM, Davis AP, Dolinski K, Dwight SS, Eppig JT, et al: Gene ontology: Tool for the unification of biology. The Gene Ontology Consortium. Nat Genet 25: 25-29, 2000

19. Croft D, O'Kelly G, Wu G, Haw R, Gillespie M, Matthews L, Caudy M, Garapati P, Gopinath G, Jassal B, et al: Reactome: A database of reactions, pathways and biological processes. Nucleic Acids Res 39 (Database Issue): D691-D697, 2011.

20. Huang da W, Sherman BT and Lempicki RA: Systematic and integrative analysis of large gene lists using DAVID bioinformatics resources. Nat Protoc 4: 44-57, 2009.

21. von Mering C, Huynen M, Jaeggi D, Schmidt S, Bork P and Snel B: STRING: A database of predicted functional associations between proteins. Nucleic Acids Res 31: 258-261, 2003.

22. Smoot ME, Ono K, Ruscheinski J, Wang PL and Ideker T: Cytoscape 2.8: New features for data integration and network visualization. Bioinformatics 27: 431-432, 2011.

23. Kou Y, Zhang S, Chen X and Hu S: Gene expression profile analysis of colorectal cancer to investigate potential mechanisms using bioinformatics. Onco Targets Ther 8: 745-752, 2015.

24. Hatfield GW, Hung SP and Baldi P: Differential analysis of DNA microarray gene expression data. Mol Microbiol 47: 871-877, 2003.

25. Pende A, Artom N, Bertolotto M, Montecucco F and Dallegri F: Role of neutrophils in atherogenesis: An update. Eur J Clin Invest 46: 252-263, 2016.

26. Lee JS and Gotlieb AI: Understanding the role of the cytoskeleton in the complex regulation of the endothelial repair. Histol Histopathol 18: 879-887, 2003.

27. Nam D and Kim SY: Gene-set approach for expression pattern analysis. Brief Bioinform 9: 189-197, 2008.

28. Ammirati E, Moroni F, Magnoni M and Camici PG: The role of $\mathrm{T}$ and $\mathrm{B}$ cells in human atherosclerosis and atherothrombosis. Clin Exp Immunol 179: 173-187, 2015.

29. Zhou Q and Liao JK: Rho kinase: An important mediator of atherosclerosis and vascular disease. Curr Pharm Des 15: 3108-3115, 2009.

30. Mazzone A, Mazzucchelli I, Fossati G, Gritti D, Girola S, Canale C, Cusa $\mathrm{C}$ and Ricevuti G: Iloprost effects on phagocytes in patients suffering from ischaemic diseases: In vivo evidence for down-regulation of alpha M beta 2 integrin. Eur J Clin Invest 26: 860-866, 1996.

31. Zirlik A, Maier C, Gerdes N, MacFarlane L, Soosairajah J, Bavendiek U, Ahrens I, Ernst S, Bassler N, Missiou A, et al: CD40 ligand mediates inflammation independently of CD40 by interaction with Mac-1. Circulation 115: 1571-1580, 2007.

32. de la Cuesta F, Zubiri I, Maroto AS, Posada M, Padial LR, Vivanco F, Alvarez-Llamas G and Barderas MG: Deregulation of smooth muscle cell cytoskeleton within the human atherosclerotic coronary media layer. J Proteomics 82: 155-165, 2013.

This work is licensed under a Creative Commons Attribution-NonCommercial-NoDerivatives 4.0 International (CC BY-NC-ND 4.0) License. 